\title{
Characterization progress of a UV-microscope recently implemented at the PTB Nanometer Comparator for uni- and bidirectional measurements
}

\author{
Jan Krüger*, Rainer Köning, and Bernd Bodermann \\ Physikalisch-Technische Bundesanstalt (PTB), Bundesallee 100, 38116 Braunschweig
}

\begin{abstract}
In this contribution, we emphasize the need for a sophisticated characterization of measurement devices in particular for optical bidirectional measurements. As an example, the ongoing characterization of the UV-microscope at PTB's linescale comparator is presented. First results of spectroscopic measurements of the employed UV-LED are presented and the impact of deviations in the center wavelength of the LED on the measurements are illustrated by rigorous simulations.
\end{abstract}

\section{Introduction}

The steadily increasing demand for precisely manufactured micro- and nanostructured objects stresses the importance of optical dimensional inspections systems. They are significantly faster than competing methods such as tactile probing, are non-destructive, and do not contaminate the sample. For dimensional inspections, unidirectional and bidirectional distance measurements must be distinguished. Distance measurements in between two arbitrary features on an object such as the pitch between two lines are described as unidirectional measurements while distances in between two opposing edges, like line widths or diameters, are referred to as bidirectional measurands. Bidirectional measurements in optical microscopy always require numerical simulations of the microscopes imaging process for further evaluations, because the measured image profiles are influenced by interference and diffraction. The simulations are necessary to locate correctly and accurately, in the measured data, the positions of the physical edges of a structure under test. Therefore, in the most common and easiest way, the intensity level at the position of the edge, subsequently referred to as threshold, is identified in the simulated profile. The transfer of the threshold to the measured line profile allows to determine the correct edge position. However, the simulations are highly dependent on the illumination and imaging parameter of the used microscope and the properties of the measurement object [1]. Hence, investigations of the microscope parameters are inevitable when accurate bidirectional measurements are desired. In section 2, the UV- microscope at PTB's linescale comparator, the socalled Nanometer Comparator is introduced. Then, first results of the characterization are presented and discussed in section 3 .

\section{Characterization setup}

The Nanometer Comparator is an interferometry-based length comparator at PTB. Until now, it is used to measure one dimensional, unidirectional distance on objects with length graduations. The Nanometer Comparator performs position measurements on objects up to a size of $550 \mathrm{~mm}$ with measurement uncertainties of some nanometers. Typical tasks of the Nanometer Comparator include calibrations of interferometers, line scales, photomasks, and encoders [2]. This field of activity is expanded by bidirectional width measurement through the implementation of a more suited and well characterized UV-microscope. The reflected light microscope originates from a commercial mask metrology tool for the inspection of photomasks. It is equipped with two objectives with a respective NA of 0.55 and 0.9 . The total magnification of the microscope is $200 \mathrm{x}$ for both objectives. Here an UV-LED is used as light source. The emission is transferred with an optic fiber to the setup. To facilitate the characterization process, the microscope was

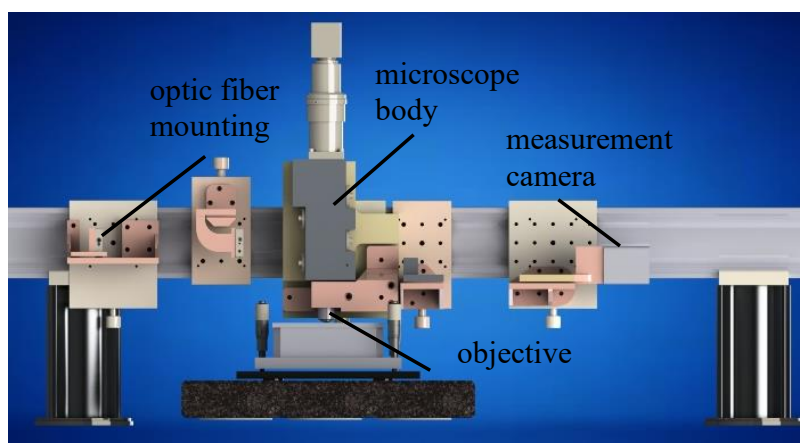

Fig. 1. Illustration of the UV-microscope in its X95 configuration

\footnotetext{
* Corresponding author: jan.krueger@ptb.de
} 
implemented in a test stand as shown in figure 1. Here, the main advantage is that the open configuration allows for the investigation of single optical planes which would be mechanically inaccessible otherwise.

\section{Characterization process}

\subsection{Overview}

For the characterization, we conduct investigations of all microscope parameters which affect the simulations. Therefore, the optical components of the microscope will be tested for their specific impact on the polarization state. Wave front deformations which may be caused by residual optical aberrations and misalignments will be determined by Shack-Hartmann sensors. Especially here, the characterization will benefit from the accessible setup since the sensor has a certain mechanical size which sometimes limits its usage. The optical aberrations obtained through the Shack-Hartmann sensor are represented in form of Zernike polynomials, which can be directly included in the simulations. Interferometric experiments concerning the coherence of the illumination source are performed, too. Here, experiments with a Michelson interferometer indicate that the coherence length of the LED is slightly enlarged by the fiber. Finally, the characterization results will be included in the simulations. The simulation results will be compared to actual microscope measurements.

\subsection{Example: UV-LED wavelength}

One important parameter is the emission wavelength of the employed LED, because temperature shifts and variable supply currents can influence the wavelength. Therefore, the spectrum of the LED, whose temperature is regulated at $29,5^{\circ} \mathrm{C}$, is measured at three different supply currents with a spectrometer. The results are shown in figure 2.

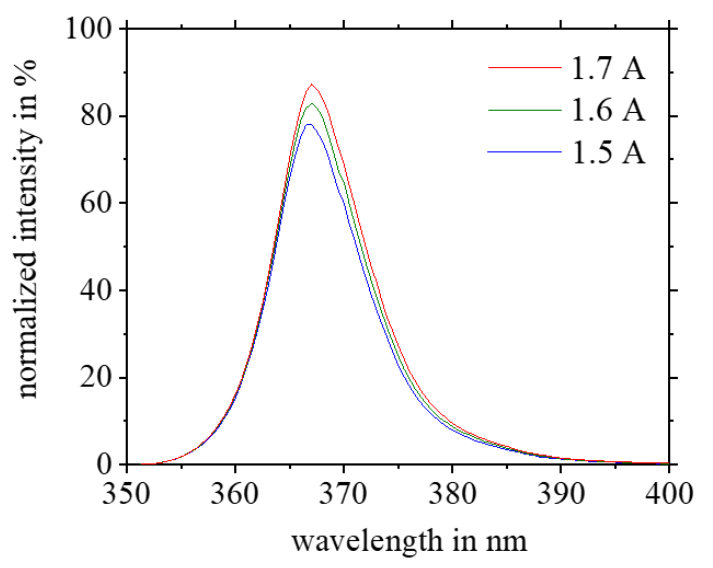

Fig. 2. LED spectrum at $29,5^{\circ} \mathrm{C}$

The center wavelength of the nominal $365 \mathrm{~nm}$ UV-LED is measured to be $367 \mathrm{~nm}$. This does not change for the different supply currents. There is only a change in normalized intensity with a linear increase with increasing supply current as expected for a LED. The importance of the knowledge of the correct operation wavelength demonstrated in the simulations shown in figure 3 . There, the image profile for one unit-cell of a binary line grating is simulated by the means of a numerical simulation tool which is based on the rigorous coupled wave analysis [3].

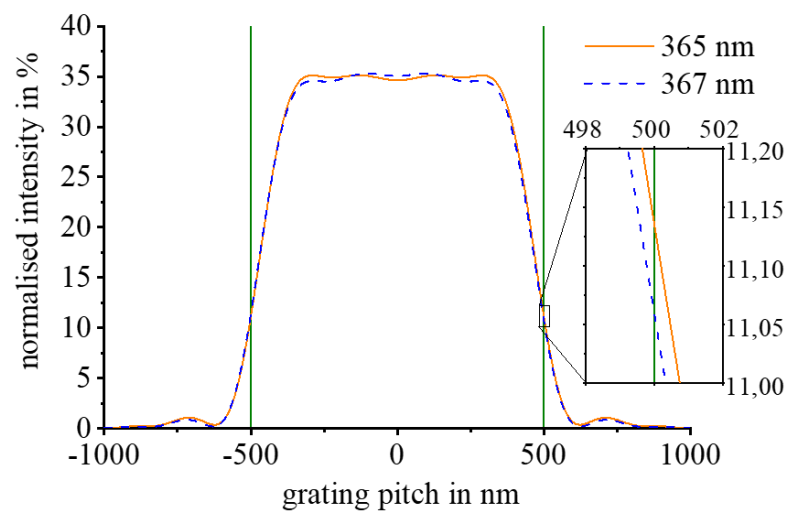

Fig. 3. Simulated profiles for two wavelengths; $\mathrm{NA}_{\text {ill. }}=\mathrm{NA}_{\text {img. }}=0,9 ;$ magnification $=200 \mathrm{x}$

The width of the unit-cell is $2 \mu \mathrm{m}$. The line consists of chrome, has a width of $1 \mu \mathrm{m}$ and a height of $100 \mathrm{~nm}$, and is applied on a glass substrate. The illumination and imaging parameters for the simulations are adapted from the microscope.

Even though, the difference between the two wavelengths is small, there are deviations between the two normalized profiles. However, they nevertheless lead to a small but measurable variation in the threshold values derived at the positions of the simulated edges indicated by two vertical lines in the figure. The thresholds at both edges are equivalent for the respective profiles since the line grating is symmetrical. A wavelength of $365 \mathrm{~nm}$ leads to a threshold value of $11.14 \%$, while a wavelength of $367 \mathrm{~nm}$ yields a threshold of $11.06 \%$. This difference in the thresholds is quite small but would already lead to a measurement error of $0.7 \mathrm{~nm}$ for both objectives, if not taken into account. In conclusion, it must be acknowledged that the wavelength is just one of many parameters which are considered in the simulation. The difference between the simulations with the assumed parameters based on manufacturer information for example and the simulations with the parameters deduced during the characterization is expected to be more significant. In the final contribution, we will further elaborate on the characterization of UV-microscope at the Nanometer Comparator and on the results shown here.

\section{References}

[1] B. Bodermann et al., Fringe 2013 (Springer-Verlag, Berlin Heidelberg, 2014)

[2] H. Bosse, G. Wilkening, Meas. Sci. Technol.16 2155 (2005)

[3] MicroSim, rigorous simulation tool for diffraction problems, developed by ITO, University of Stuttgart: https:/www.ito.uni-stuttgart.de/en/research/grouphms/microsim/ (accessed on: 18.03.2020) 